Supporting Information for:

\title{
Energy Component Analysis of Electric-Field-Induced Shape Change in Water Nanodroplets
}

\author{
Jane HyoJin Lee ${ }^{1}$, Changho Kim²*, Mayya Tokman ${ }^{2}$, and Michael E. Colvin ${ }^{3}$ \\ ${ }^{1}$ Department of Mathematics, Stonehill College, Easton, Massachusetts 02357, United States \\ ${ }^{2}$ Department of Applied Mathematics, University of California, Merced, California 95343, United States \\ ${ }^{3}$ Department of Chemistry and Biochemistry, University of California, Merced, California 95343, United States \\ * Corresponding Author \\ e-mail: ckim103@ucmerced.edu
}




\section{Section S1. Tables}

Table S1. List of stretch simulations performed. For each pair of values $\left(r_{0}, E\right), 10$ replicates were simulated except for the cases indicated by an asterisk * (for which a single run was performed). All the simulations ran for 1 ns unless indicated in the parenthesis.

\begin{tabular}{|c|c|l|}
\hline $\begin{array}{c}\text { Initial droplet } \\
\text { radius, } r_{0}[\mathrm{~nm}]\end{array}$ & Water model used & \multicolumn{1}{c|}{ Electric fields, $E[\mathrm{MV} / \mathrm{m}]$} \\
\hline 2 & SPC/E & $0,100,200,300,400,500,600,700,800(20 \mathrm{~ns}), 825(20 \mathrm{~ns}), 900,1000$ \\
\hline 2 & $\begin{array}{c}\text { SPC/E } \\
\text { (flexible) }\end{array}$ & $800,850(20 \mathrm{~ns}), 875(20 \mathrm{~ns}), 900(3 \mathrm{~ns}), 1000(2 \mathrm{~ns})$ \\
\hline 2 & $\begin{array}{c}\text { SW } \\
\text { (rigid, anisotropic) }\end{array}$ & $800(2 \mathrm{~ns}), 825,850,900(0.5 \mathrm{~ns}), 1000(0.5 \mathrm{~ns})$ \\
\hline 3 & SPC/E & $0,100,200,300,400,500,600,650(10 \mathrm{~ns}), 700(10 \mathrm{~ns}), 800,900$ \\
\hline 4 & SPC/E & $\begin{array}{l}0,100,200,300,400,500,600,612(2 \mathrm{~ns}), 619(2 \mathrm{~ns}), 622(2 \mathrm{~ns}), 625 \text { (varies from } 1 \\
\text { ns to } 2.5 \mathrm{~ns}), 650,700,800^{*}, 900^{*}\end{array}$ \\
\hline
\end{tabular}

Table S2. Simulation results of the stretch experiment. "No extension" in the column of extension time indicates that no transition to a highly extended ellipsoid was observed in any replicate simulations. Asterisks $*$ in the column of electric field indicate that only a few of the simulations showed significant shape elongation for the electric field. In the columns of shape parameter $S$, aspect ratio $\lambda$, surface area, and mean angle, the mean and standard deviation of time-averaged values computed from replicate simulations are shown. In case of no extension, the time average was performed over the whole simulation interval. Otherwise, the time average was computed over the time interval the droplet spent in an extended shape. In the columns of extension time and evaporation rate, the mean and standard deviation of sample values measured from replicate simulations are shown. Extension time was measured by the time that the shape parameter $S$ attains the mean value of the minimum and maximum of the sigmoid function fitted to the time trajectory of $S$.

\begin{tabular}{|c|c|c|c|c|c|c|c|c|}
\hline \begin{tabular}{|c|} 
Initial \\
droplet \\
radius, \\
$r_{0}[\mathrm{~nm}]$ \\
\end{tabular} & $\begin{array}{c}\text { Water } \\
\text { model } \\
\text { used }\end{array}$ & $\begin{array}{c}\text { Electric field, } E \\
{[\mathrm{MV} / \mathrm{m}]}\end{array}$ & $\begin{array}{l}\text { Extension time } \\
{[\mathrm{ps}]}\end{array}$ & Shape parameter, $S$ & $\begin{array}{c}\text { Aspect ratio, } \\
\lambda=c / a\end{array}$ & $\begin{array}{c}\text { Ellipsoid surface area } \\
\qquad\left[\AA^{2}\right]\end{array}$ & $\begin{array}{c}\text { Water evaporation } \\
\text { rate } \\
\text { [waters } / \mathrm{ns}]\end{array}$ & $\begin{array}{c}\text { Mean angle between } \\
\text { dipole and electric field } \\
\text { [degrees] }\end{array}$ \\
\hline \multirow{13}{*}{2} & \multirow{13}{*}{$\mathrm{SPC} / \mathrm{E}$} & 0 & No extension & $0.0002 \pm 0.0002$ & $0.9962 \pm 0.0164$ & $5041 \pm 13$ & $4.3 \pm 1.8$ & $90.01 \pm 0.04$ \\
\hline & & 100 & No extension & $0.0004 \pm 0.0001$ & $1.0108 \pm 0.0201$ & $5040 \pm 10$ & $5.0 \pm 2.0$ & $89.30 \pm 0.03$ \\
\hline & & 200 & No extension & $0.0004 \pm 0.0002$ & $1.0181 \pm 0.0142$ & $5040 \pm 12$ & $5.8 \pm 3.5$ & $88.60 \pm 0.05$ \\
\hline & & 300 & No extension & $0.0003 \pm 0.0002$ & $1.0431 \pm 0.0163$ & $5048 \pm 10$ & $4.6 \pm 1.8$ & $87.86 \pm 0.05$ \\
\hline & & 400 & No extension & $0.0006 \pm 0.0002$ & $1.0711 \pm 0.0192$ & $5056 \pm 11$ & $3.6 \pm 2.2$ & $87.14 \pm 0.07$ \\
\hline & & 500 & No extension & $0.0013 \pm 0.0006$ & $1.1223 \pm 0.0170$ & $5060 \pm 9$ & $5.9 \pm 1.7$ & $86.29 \pm 0.09$ \\
\hline & & 600 & No extension & $0.0037 \pm 0.0010$ & $1.1848 \pm 0.0139$ & $5077 \pm 10$ & $7.8 \pm 2.6$ & $85.36 \pm 0.04$ \\
\hline & & 700 & No extension & $0.0121 \pm 0.0012$ & $1.2786 \pm 0.0113$ & $5116 \pm 9$ & $6.4 \pm 3.0$ & $84.15 \pm 0.07$ \\
\hline & & 800 & No extension & $0.0410 \pm 0.0032$ & $1.4422 \pm 0.0119$ & $5046 \pm 21$ & $7.1 \pm 3.6$ & $82.52 \pm 0.07$ \\
\hline & & $825 *$ (4 samples) & No extension & $0.0653 \pm 0.0073$ & $1.5257 \pm 0.0202$ & $5098 \pm 13$ & $9.3 \pm 1.3$ & $81.85 \pm 0.10$ \\
\hline & & $825 *$ (6 samples) & $1208 \pm 669$ & $1.7551 \pm 0.0014$ & $8.3047 \pm 0.0253$ & $8202 \pm 41$ & $7.3 \pm 4.0$ & $49.38 \pm 0.07$ \\
\hline & & 900 & $235 \pm 73$ & $1.7876 \pm 0.0060$ & $8.9589 \pm 0.1325$ & $8773 \pm 63$ & $11.9 \pm 2.8$ & $47.31 \pm 0.31$ \\
\hline & & 1000 & $88 \pm 10$ & $1.8127 \pm 0.0024$ & $9.5726 \pm 0.0649$ & $8943 \pm 64$ & $15.6 \pm 2.3$ & $44.67 \pm 0.14$ \\
\hline \multirow{6}{*}{2} & \multirow{6}{*}{$\begin{array}{l}\mathrm{SPC} / \mathrm{E} \\
\text { (flex.) }\end{array}$} & 800 & No extension & $0.0154 \pm 0.0048$ & $1.3017 \pm 0.0314$ & $5004 \pm 14$ & $0.6 \pm 0.8$ & $83.97 \pm 0.14$ \\
\hline & & 850 & No extension & $0.0407 \pm 0.0041$ & $1.4432 \pm 0.0161$ & $5032 \pm 13$ & $1.5 \pm 1.3$ & $82.91 \pm 0.07$ \\
\hline & & $875 *$ (3 samples) & No extension & $0.0669 \pm 0.0081$ & $1.5271 \pm 0.0198$ & $5078 \pm 10$ & $1.0 \pm 1.0$ & $82.23 \pm 0.11$ \\
\hline & & $875 *(7$ samples $)$ & $5494 \pm 5219$ & $1.8445 \pm 0.0008$ & $10.5498 \pm 0.0270$ & $8907 \pm 23$ & $0.7 \pm 1.5$ & $42.01 \pm 0.12$ \\
\hline & & 900 & $2029 \pm 1960$ & $1.8485 \pm 0.0025$ & $10.6947 \pm 0.0922$ & $8969 \pm 54$ & $0.9 \pm 0.9$ & $41.55 \pm 0.21$ \\
\hline & & 1000 & $337 \pm 54$ & $1.8587 \pm 0.0022$ & $11.0913 \pm 0.0908$ & $9077 \pm 46$ & $1.6 \pm 1.2$ & $39.79 \pm 0.30$ \\
\hline \multirow{6}{*}{2} & \multirow{6}{*}{ SW } & 800 & No extension & $0.0635 \pm 0.0169$ & $1.5201 \pm 0.0484$ & $5130 \pm 38$ & $18.2 \pm 5.7$ & $82.51 \pm 0.24$ \\
\hline & & $825^{*}$ (3 samples) & No extension & $0.1459 \pm 0.0613$ & $1.7267 \pm 0.1335$ & $5278 \pm 83$ & $20.8 \pm 6.7$ & $81.04 \pm 0.87$ \\
\hline & & $825 *$ (7 samples) & $591 \pm 267$ & $1.8412 \pm 0.0031$ & $10.4442 \pm 0.1045$ & $9169 \pm 61$ & $24.0 \pm 6.7$ & $44.37 \pm 0.35$ \\
\hline & & 850 & $323 \pm 95$ & $1.8466 \pm 0.0038$ & $10.6290 \pm 0.1362$ & $9237 \pm 68$ & $30.2 \pm 5.3$ & $43.43 \pm 0.21$ \\
\hline & & 900 & $146 \pm 29$ & $1.8521 \pm 0.0054$ & $10.8373 \pm 0.2020$ & $9400 \pm 124$ & $39.4 \pm 8.3$ & $42.51 \pm 0.24$ \\
\hline & & 1000 & $89 \pm 16$ & $1.8659 \pm 0.0028$ & $11.4076 \pm 0.1237$ & $9467 \pm 168$ & $51.4 \pm 8.2$ & $40.47 \pm 0.23$ \\
\hline
\end{tabular}


Energy Component Analysis of Electric-Field-Induced Shape Change in Water Nanodroplets - Supporting Information

\begin{tabular}{|c|c|c|c|c|c|c|c|c|}
\hline \multirow{12}{*}{3} & \multirow{12}{*}{$\mathrm{SPC} / \mathrm{E}$} & 0 & No extension & $0.0000 \pm 0.0001$ & $0.9881 \pm 0.0130$ & $11309 \pm 8$ & $4.9 \pm 3.5$ & $90.00 \pm 0.02$ \\
\hline & & 100 & No extension & $0.0001 \pm 0.0001$ & $1.0050 \pm 0.0101$ & $11300 \pm 8$ & $7.4 \pm 2.8$ & $89.36 \pm 0.01$ \\
\hline & & 200 & No extension & $0.0001 \pm 0.0001$ & $1.0307 \pm 0.0144$ & $11309 \pm 10$ & $7.0 \pm 2.7$ & $88.69 \pm 0.03$ \\
\hline & & 300 & No extension & $0.0002 \pm 0.0001$ & $1.0590 \pm 0.0136$ & $11318 \pm 11$ & $6.7 \pm 2.4$ & $88.00 \pm 0.03$ \\
\hline & & 400 & No extension & $0.0007 \pm 0.0001$ & $1.1088 \pm 0.0074$ & $11333 \pm 10$ & $8.0 \pm 2.3$ & $87.23 \pm 0.03$ \\
\hline & & 500 & No extension & $0.0028 \pm 0.0007$ & $1.1700 \pm 0.0145$ & $11366 \pm 16$ & $8.3 \pm 2.1$ & $86.37 \pm 0.05$ \\
\hline & & 600 & No extension & $0.0122 \pm 0.0021$ & $1.2817 \pm 0.0153$ & $11452 \pm 18$ & $11.3 \pm 2.8$ & $85.29 \pm 0.07$ \\
\hline & & \begin{tabular}{|l|}
650 \\
\end{tabular} & No extension & $0.0296 \pm 0.0024$ & $1.3967 \pm 0.0115$ & $11470 \pm 22$ & $8.8 \pm 1.5$ & $84.45 \pm 0.04$ \\
\hline & & $700 *$ (5 samples) & No extension & $0.1017 \pm 0.0130$ & $1.6439 \pm 0.0361$ & $11726 \pm 44$ & $11.2 \pm 1.3$ & $83.01 \pm 0.15$ \\
\hline & & $700 *$ (5 samples) & $2579 \pm 2861$ & $1.8458 \pm 0.0010$ & $10.5838 \pm 0.0341$ & $19984 \pm 69$ & $10.6 \pm 1.7$ & $50.77 \pm 0.12$ \\
\hline & & \begin{tabular}{|l|}
800 \\
\end{tabular} & $202 \pm 23$ & $1.8734 \pm 0.0015$ & $11.7391 \pm 0.0710$ & $20997 \pm 128$ & $23.8 \pm 4.5$ & $47.39 \pm 0.13$ \\
\hline & & 900 & $111 \pm 10$ & $1.8872 \pm 0.0016$ & $12.4725 \pm 0.0876$ & $21464 \pm 133$ & $29.2 \pm 2.4$ & $44.84 \pm 0.08$ \\
\hline \multirow{16}{*}{4} & \multirow{16}{*}{$\mathrm{SPC} / \mathrm{E}$} & 0 & No extension & $0.0000 \pm 0.0000$ & $0.9972 \pm 0.0144$ & $20055 \pm 13$ & $11.5 \pm 4.0$ & $90.01 \pm 0.01$ \\
\hline & & 100 & No extension & $0.0000 \pm 0.0000$ & $1.0081 \pm 0.0109$ & $20051 \pm 11$ & $14.1 \pm 3.1$ & $89.37 \pm 0.02$ \\
\hline & & 200 & No extension & $0.0000 \pm 0.0000$ & $1.0332 \pm 0.0108$ & $20065 \pm 11$ & $11.6 \pm 3.6$ & $88.73 \pm 0.02$ \\
\hline & & 300 & No extension & $0.0002 \pm 0.0001$ & $1.0776 \pm 0.0081$ & $20080 \pm 12$ & $12.4 \pm 4.1$ & $88.03 \pm 0.03$ \\
\hline & & 400 & No extension & $0.0011 \pm 0.0003$ & $1.1241 \pm 0.0116$ & $20119 \pm 11$ & $16.8 \pm 4.1$ & $87.28 \pm 0.04$ \\
\hline & & 500 & No extension & $0.0068 \pm 0.0016$ & $1.2280 \pm 0.0186$ & $20236 \pm 23$ & $14.7 \pm 2.4$ & $86.33 \pm 0.04$ \\
\hline & & 600 & No extension & $0.0549 \pm 0.0142$ & $1.4879 \pm 0.0432$ & $20667 \pm 84$ & $17.7 \pm 5.1$ & $84.76 \pm 0.14$ \\
\hline & & 612 & No extension & $0.0974 \pm 0.0211$ & $1.6239 \pm 0.0479$ & $20914 \pm 114$ & $21.8 \pm 4.1$ & $84.16 \pm 0.19$ \\
\hline & & \begin{tabular}{|l|}
619 \\
\end{tabular} & No extension & $0.1533 \pm 0.0481$ & $1.7617 \pm 0.1164$ & $21195 \pm 231$ & $22.7 \pm 8.0$ & $83.61 \pm 0.42$ \\
\hline & & $622 *$ (7 samples) & No extension & $0.1306 \pm 0.0181$ & $1.7093 \pm 0.0443$ & $21093 \pm 99$ & $17.0 \pm 2.0$ & $83.76 \pm 0.16$ \\
\hline & & $622 *$ (3 samples) & $1210 \pm 40$ & $1.8810 \pm 0.0060$ & $12.1442 \pm 0.2907$ & $37257 \pm 251$ & $21.7 \pm 5.0$ & $52.60 \pm 0.19$ \\
\hline & & \begin{tabular}{|l|}
625 \\
\end{tabular} & $1299 \pm 55$ & $1.8736 \pm 0.0095$ & $11.8425 \pm 0.4354$ & $37061 \pm 558$ & $20.6 \pm 3.6$ & $52.88 \pm 0.45$ \\
\hline & & 650 & $563 \pm 7$ & $1.8904 \pm 0.0017$ & $12.7253 \pm 0.1156$ & $37851 \pm 155$ & $24.8 \pm 3.4$ & $51.27 \pm 0.09$ \\
\hline & & 700 & $268 \pm 3$ & $1.9021 \pm 0.0010$ & $13.4749 \pm 0.0878$ & $38615 \pm 136$ & $32.7 \pm 5.0$ & $49.21 \pm 0.06$ \\
\hline & & 800 (1 sample) & 160 & 1.9178 & 14.8139 & 39593 & 46 & 46.2 \\
\hline & & 900 (1 sample) & 103 & 1.9259 & 15.8378 & 420672 & 54 & 44.0 \\
\hline
\end{tabular}

Table S3. Simulation results of the collapse experiment. For each droplet size, estimated values of the critical electric field strength $E_{\text {crit }}$ determined by the collapsing experiment are given as well as the range of electric field strength used to determine $E_{\text {crit }}$.

\begin{tabular}{|c|c|c|c|}
\hline $\begin{array}{c}\text { Initial droplet radius, } \\
r_{0}[\mathrm{~nm}]\end{array}$ & $\begin{array}{c}\text { Number of water } \\
\text { molecules }\end{array}$ & $\begin{array}{c}\text { Range of electric field strength } E \\
\text { used for searching for } E_{\text {crit }}[\mathrm{MV} / \mathrm{m}]\end{array}$ & $\begin{array}{c}\text { Estimated critical field strength, } \\
E_{\text {crit }}[\mathrm{MV} / \mathrm{m}]\end{array}$ \\
\hline 1.45 & 426 & $888-891$ & 891 \\
\hline 1.53 & 499 & $863-866$ & 865 \\
\hline 1.63 & 605 & $800-827$ & 807 \\
\hline 1.79 & 806 & $780-791$ & 790 \\
\hline 1.92 & 985 & $738-758$ & 758 \\
\hline 2.17 & 1422 & $700-705$ & 705 \\
\hline 2.41 & 1950 & $661-666$ & 666 \\
\hline 2.64 & 2566 & $625-635$ & 634 \\
\hline 2.87 & 3298 & $602-616$ & 605 \\
\hline 3.09 & 4128 & $580-591$ & 583 \\
\hline 3.30 & 4991 & $550-558$ & 558 \\
\hline 3.48 & 5863 & $538-541$ & 539 \\
\hline 3.66 & 6827 & $525-528$ & 526 \\
\hline 3.83 & 7835 & $512-515$ & 513 \\
\hline 4.00 & 8932 & $500-503$ & 501 \\
\hline
\end{tabular}


Table S4. Simulation results of the energy cycle analysis. Estimated values of energy components and relevant physical quantities are shown for each state in the energy cycle. State A is a spherical shape with dipoles not aligned $(E=0)$, State $\mathrm{B}$ is a hypothetical extended shape with dipoles not aligned $(E=0)$, State $\mathrm{C}$ is an extended shape with dipoles aligned $(E>0)$, and State D is a hypothetical spherical shape with dipoles aligned $(E>0)$. Note that $U_{\text {pot }}=U_{\mathrm{d}-\mathrm{f}}+U_{\text {elec }}+U_{\mathrm{LJ}}$.

\begin{tabular}{|c|c|c|c|c|c|c|c|c|}
\hline State & $\begin{array}{c}\text { Number } \\
\text { of water } \\
\text { molecules }\end{array}$ & $\begin{array}{c}\text { Dipole } \\
\text { angle } \\
{[\text { degree }]}\end{array}$ & $\begin{array}{c}\text { Dipole-field } \\
\text { interaction } \\
\text { energy, } U_{\mathrm{d}-\mathrm{f}} \\
{[\mathrm{kJ} / \mathrm{mol}]}\end{array}$ & $\begin{array}{c}\text { Dipole-dipole } \\
\text { interaction } \\
\text { energy, } U_{\mathrm{d}-\mathrm{d}} \\
{[\mathrm{kJ} / \mathrm{mol}]}\end{array}$ & $\begin{array}{c}\text { Electrostatic } \\
\text { interaction } \\
\text { energy, } U_{\mathrm{elec}} \\
{[\mathrm{kJ} / \mathrm{mol}]}\end{array}$ & $\begin{array}{c}\text { LJ interaction } \\
\text { energy, } U_{\mathrm{LJ}} \\
{[\mathrm{kJ} / \mathrm{mol}]}\end{array}$ & $\begin{array}{c}\text { Total potential } \\
\text { energy, } U_{\mathrm{pot}} \\
{[\mathrm{kJ} / \mathrm{mol}]}\end{array}$ & $\begin{array}{c}\text { Surface } \\
\text { energy, } U_{\text {surf }} \\
{[\mathrm{kJ} / \mathrm{mol}]}\end{array}$ \\
\hline $\mathrm{A}$ & $8924 \pm 4$ & $90.1 \pm 0.1$ & 0 & $-216235 \pm 580$ & $-483442 \pm 879$ & $78414 \pm 620$ & $-405008 \pm 369$ & $14864 \pm 27$ \\
\hline $\mathrm{B}$ & $8890 \pm 14$ & $80.1 \pm 0.6$ & 0 & $-205695 \pm 249$ & $-446755 \pm 1001$ & $76500 \pm 617$ & $-370256 \pm 788$ & $27867 \pm 492$ \\
\hline $\mathrm{C}$ & $8890 \pm 14$ & $52.2 \pm 0.6$ & $-16082 \pm 218$ & $-214834 \pm 478$ & $-466196 \pm 845$ & $76580 \pm 620$ & $-405698 \pm 343$ & $27867 \pm 492$ \\
\hline $\mathrm{D}$ & $8924 \pm 4$ & $51.3 \pm 0.7$ & $-16464 \pm 252$ & $-128261 \pm 1089$ & $-346326 \pm 1093$ & $78414 \pm 620$ & $-284376 \pm 767$ & $14864 \pm 27$ \\
\hline
\end{tabular}

Table S5. Fitting parameter values for the total potential energy function $U_{\text {pot }}(\lambda, E)$. Note that we assume $U_{\text {pot }}(\lambda, E)=U_{\mathrm{d}-\mathrm{f}}(\lambda, E)+U_{\text {int }}(\lambda)=-\mu E \Theta(\lambda)+U_{\text {int }}(\lambda)$ with $\Theta(\lambda)=a_{4} \lambda^{4}+a_{3} \lambda^{3}+a_{2} \lambda^{2}+a_{1} \lambda+a_{0}$ and $U_{\text {int }}(\lambda)=b \lambda^{c}$.

\begin{tabular}{|c|c|c|c|c|}
\hline & Parameters & $r_{0}=2 \mathrm{~nm}$ & $r_{0}=3 \mathrm{~nm}$ & $r_{0}=4 \mathrm{~nm}$ \\
\hline \multirow{4}{*}{$\Theta$} & $a_{4}$ & $-2.1017 \times 10^{-1}$ & $-1.8393 \times 10^{-1}$ & $-1.3469 \times 10^{-1}$ \\
\cline { 2 - 5 } & $a_{3}$ & $5.3233 \times 10^{0}$ & $6.6051 \times 10^{0}$ & $5.9023 \times 10^{0}$ \\
\cline { 2 - 5 } & $a_{2}$ & $-5.4726 \times 10^{1}$ & $-9.8488 \times 10^{1}$ & $-1.1685 \times 10^{2}$ \\
\cline { 2 - 5 } & $a_{1}$ & $3.2519 \times 10^{2}$ & $8.1554 \times 10^{2}$ & $1.3274 \times 10^{3}$ \\
\cline { 2 - 5 } & $a_{0}$ & $-2.4055 \times 10^{2}$ & $-6.3213 \times 10^{2}$ & $-1.0032 \times 10^{3}$ \\
\hline \multirow{3}{*}{$U_{\text {int }}$} & $b[\mathrm{~kJ} / \mathrm{mol}]$ & $4.8881 \times 10^{4}$ & $1.6980 \times 10^{5}$ & $4.0537 \times 10^{5}$ \\
\cline { 2 - 5 } & $c$ & $-2.8678 \times 10^{-2}$ & $-2.1700 \times 10^{-2}$ & $-1.6058 \times 10^{-2}$ \\
\hline
\end{tabular}




\section{Section S2. Analysis Methods}

To analyze the structural and energetic properties of the droplets in the electric fields, we used tools from the Gromacs package as well as self-written programs. The $x, y$, and $z$ coordinates of the droplet were sampled every 10 ps from the production simulation trajectories to calculate the nanodroplet properties.

\section{$\underline{\text { S2.1. Structural Analysis }}$}

Evaporating water. Throughout the simulation we monitored the number of water molecules evaporated from the droplet. Water molecules were defined as "evaporated" using the same algorithm we used for creating the initial structures after equilibration (see manuscript).

Shape related parameters. To study the relationship between the shape of the droplet and the electric field strength, we quantified the droplet shape by a scalar number called the shape parameter. This shape measuring technique is commonly used to analyze conformations of proteins ${ }^{1}$, or polymers ${ }^{2}$. Characteristics of the conformation such as asphericity, acylindricity, and anisotropy can be measured using the gyration tensor ${ }^{3}$ which describes the second moments ${ }^{4}$ of the position of a collection of particles.

The gyration tensor, $T$, in a Cartesian coordinate system is expressed as

$$
T=\frac{1}{N} \sum_{i=1}^{N}\left[\begin{array}{ccc}
r_{x}^{i} r_{x}^{i} & r_{x}^{i} r_{y}^{i} & r_{x}^{i} r_{z}^{i} \\
r_{y}^{i} r_{x}^{i} & r_{y}^{i} r_{y}^{i} & r_{y}^{i} r_{z}^{i} \\
r_{z}^{i} r_{x}^{i} & r_{z}^{i} r_{y}^{i} & r_{z}^{i} r_{z}^{i}
\end{array}\right]
$$

where a position vector $r^{i}$ is the displacement of the $i^{\text {th }}$ particle from the center of mass of a droplet. In the computation, only the coordinates of the oxygen atoms $x, y$, and $z$ are used to produce the gyration tensor matrix. Since the mass, $m$ of $N$ oxygen atoms are the same, coordinates of the center of mass of the droplet are simplified as

$$
r_{\mathrm{CM}}=\frac{\sum_{i=1}^{N} r^{i} m^{i}}{\sum_{i=1}^{N} m^{i}}=\frac{1}{N} \sum_{i=1}^{N} r^{i}
$$

Hence, for the droplet in a three-dimensional Cartesian coordinate system, the gyration tensor becomes

$$
T=\frac{1}{N} \sum_{i=1}^{N}\left[\begin{array}{ccc}
\overline{\left(x^{i}-x_{\mathrm{CM}}^{i}\right)^{2}} & \overline{\left(x^{i}-x_{\mathrm{CM}}^{i}\right)\left(y^{i}-y_{\mathrm{CM}}^{i}\right)} & \overline{\left(x^{i}-x_{\mathrm{CM}}^{i}\right)\left(z^{i}-z_{\mathrm{CM}}^{i}\right)} \\
\frac{\left.y_{\mathrm{CM}}^{i}\right)\left(x^{i}-x_{\mathrm{CM}}^{i}\right)}{\left(z^{i}-z_{\mathrm{CM}}^{i}\right)\left(y^{i}-y_{\mathrm{CM}}^{i}\right)} & \frac{\left(y^{i}-y_{\mathrm{CM}}^{i}\right)^{2}}{\left(z^{i}-z_{\mathrm{CM}}^{i}\right)\left(y^{i}-y_{\mathrm{CM}}^{i}\right)} & \frac{\left(y^{i}-y_{\mathrm{CM}}^{i}\right)\left(z^{i}-z_{\mathrm{CM}}^{i}\right)}{\left(z^{i}-z_{\mathrm{CM}}^{i}\right)^{2}}
\end{array}\right] .
$$

The eigenvalues of the gyration tensor, $T$, called the principal moments, are used to compute asphericity $A$ and the shape parameter $S$ of the $\operatorname{droplet}^{1}$ defined as

$$
A=\frac{\sum_{i=1}^{3}\left(\lambda_{i}-\bar{\lambda}\right)^{2}}{6(\bar{\lambda})^{2}}, \quad S=\frac{\prod_{i=1}^{3}\left(\lambda_{i}-\bar{\lambda}\right)}{(\bar{\lambda})^{3}}
$$


where $\lambda_{i}$ is the $i^{\text {th }}$ eigenvalue of $T$ and $\bar{\lambda}$ is the average of the eigenvalues. The asphericity parameter $A$ is always non-negative and zero when particles are distributed in a spherically symmetric way (i.e. sphere). The shape parameter $S$ is always bounded between $-1 / 4$ and 2 and the range of $S$ values corresponds to the shapes of the ellipsoid. The shape is defined to be an oblate ellipsoid for $-1 / 4<S<0$, a sphere for $S=0$, and a prolate ellipsoid for $0<S<2$.

Dipole Alignment. In order to understand the mechanism governing shape extension, we inspected the behavior of water dipoles by calculating the average angle between each dipole and the electric field direction, $z$. The average angle indicates the degree to which dipoles are aligning with the electric field as the shape is changing.

Ellipsoid Fitting. We assumed that the shape of a droplet fits an ellipsoid and computed its three principal axes and its length using the inertia tensor ${ }^{5}$. The inertia tensor $I$ is the scaling factor between angular momentum $L$ and angular velocity $\omega$ (i.e. $L=I \cdot \omega$ ), which is expressed in a Cartesian coordinate system as

$$
I=\sum_{i=1}^{N}\left[\begin{array}{ccc}
m^{i}\left[\left(r_{y}^{i}\right)^{2}+\left(r_{z}^{i}\right)^{2}\right] & -m^{i} r_{x}^{i} r_{y}^{i} & -m^{i} r_{x}^{i} r_{z}^{i} \\
-m^{i} r_{y}^{i} r_{x}^{i} & m^{i}\left[\left(r_{x}^{i}\right)^{2}+\left(r_{z}^{i}\right)^{2}\right] & -m^{i} r_{y}^{i} r_{z}^{i} \\
-m^{i} r_{z}^{i} r_{x}^{i} & -m^{i} r_{z}^{i} r_{y}^{i} & m^{i}\left[\left(r_{x}^{i}\right)^{2}+\left(r_{y}^{i}\right)^{2}\right]
\end{array}\right],
$$

where $m$ is the mass of oxygen and a position vector $r^{i}$ is again the displacement of the $i^{\text {th }}$ particle from the center of mass of a droplet. The eigenvectors of the inertia tensor represent directions of the three principal axes and the corresponding eigenvalues are called the principal moments of inertia about the given axes.

We computed the lengths of the three axes by equating the eigenvalues of the computed inertia tensor and using the moment of inertia tensor ${ }^{6}$ for solid ellipsoid with axes $a, b$, and $c$ with mass $m$, given below

$$
I=\left[\begin{array}{ccc}
\frac{1}{5} m\left(b^{2}+c^{2}\right) & 0 & 0 \\
0 & \frac{1}{5} m\left(a^{2}+c^{2}\right) & 0 \\
0 & 0 & \frac{1}{5} m\left(a^{2}+b^{2}\right)
\end{array}\right] .
$$

Surface Area. Using the axes information obtained, we calculated the approximate surface area of the droplet fitted by an ellipsoid. The surface area of a general ellipsoid ${ }^{7}$ is given by

$$
\mathrm{SA}=2 \pi c^{2}+\frac{2 \pi a b}{\sin \phi}\left[E(\phi, k) \sin ^{2} \phi+F(\phi, k) \cos ^{2} \phi\right],
$$

where three axes $a, b$, and $c$ are in order of $a \geq b \geq c$ and $\cos \phi=c / a$ and $k^{2}=a^{2}\left(b^{2}-c^{2}\right) / b^{2}\left(a^{2}-c^{2}\right)$. Two functions $F(\phi, k)$ and $E(\phi, k)$ are incomplete elliptic integrals of the first and second kind ${ }^{8}$, respectively. We used C code provided in Mathematics Source Library ${ }^{9}$ for the calculation of elliptic integrals. 


\section{$\underline{\text { S2.2. Energetic Analysis }}$}

For the energetic analysis we calculated the total potential energy comprised of three terms: (1) the electrostatic interaction energy between the water molecules, (2) the Lennard-Jones interaction energy between the water molecules, and (3) the interaction energy between the water dipoles and the applied external electric field.

The combination of electrostatic and Lennard-Jones potential energies between two water molecules A and B is given by

$$
U_{\mathrm{AB}}\left(\mathbf{r}_{\mathrm{ij}}\right)=\sum_{i=1}^{3} \sum_{j=1}^{3}\left\{\left(\frac{1}{4 \pi \varepsilon_{0}} \frac{\mathrm{q}_{\mathrm{i}} \mathrm{q}_{\mathrm{j}}}{\left\|\mathbf{r}_{\mathrm{ij}}\right\|}\right)+4 \varepsilon_{\mathrm{ij}}\left[\left(\frac{\sigma_{\mathrm{ij}}}{\left\|\mathbf{r}_{\mathrm{ij}}\right\|}\right)^{12}-\left(\frac{\sigma_{\mathrm{ij}}}{\left\|\mathbf{r}_{\mathrm{ij}}\right\|}\right)^{6}\right]\right\},
$$

where the indices $i$ and $j$ run over the three atoms, $\mathrm{H}, \mathrm{O}, \mathrm{H}$, comprising the water molecule $\mathrm{A}$ and $\mathrm{B}$, respectively. The electrostatic term is Coulomb's law with $\varepsilon_{0}$ as the vacuum permittivity, $\left(4 \pi \varepsilon_{0}\right)^{-1}=$ $138.935485 \mathrm{~kJ} \mathrm{~mol}^{-1} \mathrm{e}^{-2}$, the atom charges $\mathrm{q}_{\mathrm{i}}$ and $\mathrm{q}_{\mathrm{j}}$ and with $\left\|\mathrm{r}_{\mathrm{ij}}\right\|$ representing the distance between the atoms in A and B water molecules. The total energy of the system is the sum of the potential interaction energies between all the molecular pairs in the droplet. The first and the second terms are the Lennard-Jones interaction with parameters $\varepsilon$ (depth of the potential well) and $\sigma$ (finite distance at which the inter-particle potential is zero) depending on the types of the atoms in a pair. However, for a simple water model in MD simulation, only oxygenoxygen pairs are considered for Lennard-Jones potential. Hence, the Lennard-Jones terms for two water molecules A and B become simply

$$
\frac{C_{12}}{\left\|\mathbf{r}_{\text {oo }}\right\|^{12}}-\frac{C_{6}}{\left\|\mathbf{r}_{\text {oo }}\right\|^{6}}
$$

where $\left\|\mathbf{r}_{\mathrm{oo}}\right\|$ is the distance between oxygens of $\mathrm{A}$ and $\mathrm{B}$, and the constants are $C_{12}=4 \varepsilon_{\mathrm{oo}} \sigma_{\mathrm{oo}}{ }^{12}=$ $0.74158 \times 10^{-6} \mathrm{~kJ} \mathrm{~nm}^{12} \mathrm{~mol}^{-1}$ and $C_{6}=4 \varepsilon_{\mathrm{oo}} \sigma_{\mathrm{oo}}{ }^{6}=0.22617 \times 10^{-2} \mathrm{~kJ} \mathrm{~nm}^{6} \mathrm{~mol}^{-1}$.

The interaction energy between water dipoles and applied external electric field, $E$ is given by

$$
U_{\mu}=-\boldsymbol{\mu} \cdot \boldsymbol{E}=-|\boldsymbol{\mu}||\boldsymbol{E}| \cos \theta,
$$

where $\boldsymbol{\mu}$ is the dipole moment and $\boldsymbol{E}$ is the electric field in vector form with the conversion factor of $1 \mathrm{~kJ} \mathrm{~nm} \mathrm{~mol}{ }^{-1} \mathrm{e}^{-1}=1.0364272 \times 10^{4} \mathrm{MV} / \mathrm{m}$ and $\theta$ is the angle between the electric field direction and the dipole moment. Since we applied the electric field in the $+Z$ direction, the calculation simply reduced to the product of $Z$ component of dipole moment and the magnitude of the electric field.

In addition to the above calculation of the total potential energy, we extracted dipole-dipole interaction energy

$$
U_{\mu_{A} \mu_{B}}=\frac{1}{4 \pi \varepsilon_{0}} \frac{\left\|\mathbf{R}_{A B}\right\|^{2}\left(\mu_{A} \cdot \mu_{B}\right)-3\left(\mu_{A} \cdot \mathbf{R}_{A B}\right)\left(\mu_{B} \cdot \mathbf{R}_{A B}\right)}{\left\|\mathbf{R}_{A B}\right\|^{5}},
$$

where $\mu_{A}$ and $\mu_{B}$ are the dipole moments of two water molecules and $\mathbf{R}_{A B}$ is the vector between them.

Finally, the surface energy was computed by multiplying the surface area of the droplet and the surface enthalpy value $123 \mathrm{~mJ} / \mathrm{m}^{2}$, which is estimated from the surface tension values of SPC/E water at different temperatures ${ }^{10}$. 


\section{References}

(1) Dima, R. I.; Thirumalai, D. Asymmetry in the Shapes of Folded and Denatured States of Proteins. $J$. Phys. Chem. B 2004, 108 (21), 6564-6570. https://doi.org/10.1021/jp037128y.

(2) Theodorou, D. N.; Suter, U. W. Shape of Unperturbed Linear Polymers: Polypropylene. Macromolecules 1985, 18 (6), 1206-1214. https://doi.org/10.1021/ma00148a028.

(3) Wikipedia. Gyration tensor. https://en.wikipedia.org/wiki/Gyration_tensor.

(4) Wikipedia. Moment (mathematics). https://en.wikipedia.org/wiki/Moment_(mathematics).

(5) Baraff, D. An Introduction to Physically Based Modeling: Rigid Body Simulation I - Unconstrained Rigid Body Dynamics; 1997.

(6) Wikipedia. List of moments of inertia. http://en.wikipedia.org/wiki/List_of_moments_of_inertia.

(7) Wikipedia. Ellipsoid. https://en.wikipedia.org/wiki/Ellipsoid.

(8) Wikipedia. Elliptic integral. https://en.wikipedia.org/wiki/Elliptic_integral.

(9) Mathematics Source Library. Elliptic Integrals. http://www.mymathlib.com/functions/elliptic_integr.

(10) Vega, C.; de Miguel, E. Surface Tension of the Most Popular Models of Water by Using the Test-Area Simulation Method. J. Chem. Phys. 2007, 126 (15), 154707. https://doi.org/10.1063/1.2715577. 